Published in final edited form as:

J Am Geriatr Soc. 2017 July ; 65(7): 1554-1558. doi:10.1111/jgs.14832.

\title{
Oral Health and Dental Care in Older Asian Americans in Central Texas
}

Yuri Jang, $\mathrm{PhD}^{\star}$, Hyunwoo Yoon, $\mathrm{PhD}^{\dagger}$, Nan Sook Park, PhD ${ }^{\ddagger}$, and David A. Chiriboga, PhD§

*School of Social Work, University of Texas at Austin, Austin

tSchool of Social Work, Texas State University, San Marcos, Texas

¥School of Social Work, University of South Florida, Tampa

$\S$ Department of Child and Family Studies, University of South Florida, Tampa, Florida

\begin{abstract}
Objectives-To examine factors associated with dental health insurance, self-rated oral health, and use of preventive dental care services in older Asian Americans. DESIGN: Cross-sectional survey.
\end{abstract}

Setting-The Asian American Quality of Life Survey was conducted with 2,614 Asian Americans living in central Texas using questionnaires available in English and six Asian languages.

Participants-Asian American Quality of Life Survey participants aged 60 and older ( $\mathrm{N}=533$; mean age $=69.4 \pm 6.9$ ).

Measurements-Participants were asked whether they had insurance that covered the cost of any dental visit, how they would rate their overall oral health status, and whether they had visited a dental clinic for a routine examination in the past 12 months. Information was also collected on sociodemographic and immigration-related variables.

Results-More than $61 \%$ of the sample had no dental health insurance, $45 \%$ reported that their oral health was fair or poor, and $44 \%$ had not used preventive dental care services. A series of logistic regression analyses identified factors posing a significant risk to oral health and dental care. For example, those with limited English proficiency were 3.5 times as likely to lack dental health insurance and 3.2 times as likely to rate their oral health as fair or poor. The odds of not using preventive dental care services were 6.4 times as great in those without dental health insurance.

Conclusion-The overall findings call attention to efforts to promote oral health and dental care in older Asian Americans.

Address correspondence to Yuri Jang, School of Social Work, The University of Texas at Austin, 1925 San Jacinto Blvd., D 3500, Austin, TX 78712.yjang12@austin.utexas.edu.

Author Contributions: Jang: Concept, design, data analysis, preparation of manuscript. Yoon: Concept, design, preparation of manuscript. Park: Preparation of manuscript. Chiriboga: Concept, preparation of manuscript.

Sponsor's Role: None. 


\section{Keywords}

oral health; dental care; older Asian Americans

Ranging from the basic ability to eat to mortality, oral health affects multiple aspects of an individual's life and serves as an integral part of health and well-being. ${ }^{1-5}$ Nonetheless, many segments of the U.S. population experience inequality in oral health and dental care. ${ }^{1-3}$ Such disparity is more pronounced in elderly adults ${ }^{6,7}$ and racial and ethnic minorities. ${ }^{8-10}$ For example, older blacks and Hispanics in a nationally representative sample had poorer oral health and used dental care services less frequently than their agecomparable white counterparts. ${ }^{11}$ A similar pattern was observed in a community-based sample in which older blacks and American Indians fared worse than older whites in various oral health measures even after adjustment for socioeconomic status. ${ }^{12,13}$

With regard to older Asian Americans, there is not only a marked paucity of information, but also a problem with the generalizability of existing findings. The reports based on national surveys suggest that Asian Americans have better outcomes than other racial and ethnic groups in health and healthcare in general ${ }^{14,15}$ and specifically in oral health and dental care ${ }^{15,16}$ However, the representation of Asian Americans as a "model minority" may be misleading because national surveys include only those who speak English. ${ }^{14}$ Considering that a substantial proportion of Asian Americans are foreign-born immigrants with limited English proficiency, ${ }^{17-21}$ their oral health and dental care should be examined using a sample that reflects their cultural and linguistic diversity.

Focusing on older Asian Americans as a target population and using a sample collected using culturally and linguistically sensitive methods, the goal of the present study was to explore factors associated with three oral health-related outcomes: dental health insurance, self-rated oral health, and use of preventive dental care services.

\section{Methods}

\section{Data Set}

Data were used from the 533 participants aged 60 and older who were part of the Asian American Quality of Life (AAQoL) survey $(\mathrm{N}=2,614)$. The survey is part of the Austin, Texas's AAQoL initiative, which was in response to the rapid growth of the Asian-American population. ${ }^{22}$ Self-identified Asian Americans aged 18 and older living in the Austin area were eligible to participate.

The 10-page questionnaire was originally developed in English and then translated into six Asian languages (Chinese, Vietnamese, Korean, Hindi, Gujarati, Tagalog). Surveys were conducted using a paper-and-pencil questionnaire in participants' preferred language. Although the survey was designed to be self-administered, trained bilingual research assistants were available at each survey site for recruitment and assistance with survey administration. Seventy-six survey sessions took place at various sites across Austin (e.g., churches, temples, grocery markets, small group meetings, cultural events) from August to December 2015. The project was publicized through media and ethnic community sources, 
and referrals for individuals, groups, and organizations were actively sought. It took approximately 20 minutes to complete the 10-page questionnaire, and respondents were each paid $\$ 10$ for participation. The University of Texas at Austin's institutional review board approved the project.

\section{Measures}

Outcome Variables-The three outcome measures used in the analyses were dental health insurance, self-rated oral health, and use of preventive dental care services. Participants were asked whether they had insurance that covered the cost of any dental visit. Responses were coded as yes (0) or no (1). Self-rated oral health was assessed by asking, "How would you rate your overall oral health status?" The original 5-point responses were dichotomized into excellent, very good, good (0) versus fair or poor (1). This single-item measure has shown to be highly correlated with the results of clinical examination and has often been used as a binary index. ${ }^{11,12}$ Participants were also asked whether they had visited dental clinics for a routine examination in the past 12 months. Responses were coded as yes (0) or no (1).

Sociodemographic Variables-Sociodemographic information included age $(0=60$ $74,1=275)$, sex $(0=$ male, $1=$ female $)$, ethnic origin $(0=$ Chinese, $1=$ Asian Indian, $2=$ Korean, $3=$ Vietnamese, $4=$ other Asian $)$, marital status $(0=$ married, $1=$ not married $)$, education $(0=\geq$ high school graduation, $1=<$ high school graduation $)$, full time employment $(0=$ yes, $1=$ no $)$, and perceived financial status $(0=$ able to make ends meet, 1 $=$ not able to make ends meet).

Immigration-Related Variables-Considering the $10^{\text {th }}$ year after immigration as a marker of adaptation, ${ }^{2,3}$ length of stay in the United States was dichotomized $(0=\geq 10$ years, $1=<10$ years). English proficiency was assessed with a question about how well the respondent spoke English, using a 4-point response scale ranging from not at all to very well. Using the U.S. Census criteria, ${ }^{24}$ those who reported that they spoke English less than very well were categorized as a group with limited English proficiency $(0=$ English proficiency, 1 = limited English proficiency).

\section{Analytical Strategy}

After reviewing descriptive characteristics of the sample and bivariate correlations between study variables, logistic regression analyses of the three outcomes were performed. Using a sequential design, each outcome variable was entered as an additional predictor in the subsequent model. To be specific, the model of dental health insurance was tested only with background variables, including sociodemographic (e.g., age, sex, ethnicity, marital status, education, employment, perceived financial status) and immigration-related (e.g., length of stay in United States, English proficiency) variables. The next model of self-rated oral health included dental health insurance in addition to background variables. Finally, the model of preventive dental care service use was estimated with background variables, dental health insurance, and self-rated oral health. All analyses were performed using SPSS version 24 (IBM Corp., Armonk, NY). 


\section{Results}

\section{Descriptive Characteristics of the Sample}

Sample characteristics are summarized in Table 1. The mean age of the sample was 69.4 \pm 6.9 (range 60-98; 44\% > 75), and 55.4\% were female. The sample included 162 Chinese (30.4\%), 98 Asian Indians (18.4\%), 98 Koreans (18.4\%), 114 Vietnamese (21.4\%), and 61 individuals from other Asian groups (11.4\%). Examples of the ethnicities that participants specified in the "other" group were Filipino, Nepalese, Pakistani, Cambodian, and Japanese. Approximately one-quarter of participants were not married. More than one-third had received less than a high school education, and approximately $14 \%$ were employed full time. Approximately $30 \%$ reported financial insecurity.

Mean length of stay in the United States was $24.8 \pm 14.9$ years (range $0.5-78$ years, $20 \%<$ 10 years). Approximately $84 \%$ of the sample reported that they spoke English less than very well, and approximately $78 \%$ used non-English versions of the survey questionnaire.

With regard to oral health and dental care outcomes, $61 \%$ of the sample had no dental insurance, $45 \%$ reported that their oral health was fair or poor, and $44 \%$ had not had a dental examination in the past 12 months.

\section{Logistic Regression Models of Oral Health and Dental Care}

At the bivariate level, study variables were correlated in the expected direction (data not shown). All Spearman correlation coefficients were less than 0.45 , and no concern about collinearity was identified. The results from the series of logistic regression models are presented in Table 2. In the models of dental health insurance and self-rated oral health, common predictors included perceived financial status and English proficiency; those who reported unmet financial needs and those with limited English proficiency had higher odds of lacking dental health insurance and rating their oral health as poor or fair. In the models of dental health insurance and use of preventive dental care services, length of stay in the United States was a common predictor; those who had a shorter stay ( $<10$ years) were 5.9 times as likely to lack dental health insurance and 4.0 times as likely not to use preventive dental care.

Other associations were specific to the type of oral health and dental care outcomes. The odds of lacking dental health insurance were 1.6 times as high in those aged 75 and older and 2.1 times as high as in those who were not employed full time. Those with less than a high school education were $66 \%$ more likely to report poor or fair oral health. Using Chinese as the reference group, Asian Indians were 2.5 times as likely to lack dental health insurance, and Vietnamese were $62 \%$ less likely to rate their oral health as poor or fair. Those without dental insurance coverage were 6.4 times as likely not to use preventive dental care services.

\section{Discussion}

The present investigation was in response to the paucity of information on oral health and dental care in older Asian Americans ${ }^{15,16}$ and the lack of data that reflect the unique cultural 
and linguistic characteristics of the target population. ${ }^{14,17,18}$ It is alarming that what is currently known about Asian Americans is primarily based on national surveys that include only English-speaking individuals. ${ }^{14}$ The present study used culturally and linguistically sensitive approaches to reach out to diverse groups of Asian Americans. The strategies included not only providing Asian-language versions of the survey questionnaire, but also using research personnel (e.g., recruiters, survey assistants) who shared the languages and cultures of the target population. Furthermore, a strong partnership between the research team and key individuals and organizations within ethnic communities facilitated recruitment and participation of community members. The fact that $78 \%$ of the sample used non-English versions of the survey questionnaire and that $84 \%$ reported that they spoke English less than very well indicates that the approach enabled many older Asian Americans who are conventionally underrepresented in national surveys to be included.

Findings from the present study dispelled the notion of the "model minority" by demonstrating the heightened vulnerabilities of older Asian Americans. The rates of no dental health insurance (61.3\%) and no use of preventive dental care services (44.5\%) observed in the present sample are higher than those reported in the national adult samples of Asian Americans (38.1\% and 38.8\%, respectively). ${ }^{16}$ This discrepancy is attributable to the differences in survey methods, with one systematically excluding non-English-speaking persons and the other enabling them to participate by offering questionnaires in their native languages. When the present sample was divided according to English proficiency, the figures in the English-proficient group (31.8\% with no dental health insurance, $29.8 \%$ with no use of preventive dental care services) are close to those reported from national surveys. These findings lend support to the hypothesized upward selection in the national samples of Asian Americans and caution against the generalization of findings in such studies.

Moving beyond the frequently reported finding of a pervasive effect of socioeconomic disadvantage on health and healthcare, this study sheds light on the role of immigrationspecific contextual factors as sources of disparities. The vulnerabilities seen in newly arrived older immigrants and those with cultural and linguistic barriers should be taken into consideration in developing and implementing targeted interventions and health planning.

The significant contribution of dental health insurance coverage to predicting the use of preventive dental care services is worthy of attention. Although health insurance is a critical enabler for accessing all types of health services for all age groups, ${ }^{14-16}$ dental insurance coverage in the elderly immigrant population is a particular concern. Generally, many older adults lose employment-based dental insurance at retirement, and public insurance programs do not offer comprehensive dental coverage. ${ }^{14,15}$ For example, Medicare does not cover routine dental care, and Medicaid provides limited dental coverage for eligible individuals in only a few states. ${ }^{25}$ Texas and New York are two of the few states that offer dental care coverage for elderly Medicaid enrollees, ${ }^{26}$ but because of the welfare reforms that bar newly arrived immigrants from receiving federal benefits, many older immigrants are not eligible for such programs. Furthermore, studies conducted in Texas and New York have found that many elderly Asian Medicaid enrollees are not aware of their dental coverage, ${ }^{19,20}$ calling attention to the urgent need to educate eligible individuals on their benefits. The overall findings, that dental insurance is a driving force for the use of preventive dental care services 
but that a substantial proportion of older Asian Americans lack such coverage, underscore the importance of increasing access to dental care for uninsured and underserved individuals.

Some limitations of the present study should be noted. It was limited by its cross-sectional design and a nonrepresentative, regionally defined sample. By using culturally and linguistically sensitive approaches, the present sample included many hard-to-reach individuals, although their representativeness is not guaranteed because random selection was not possible. Caution should be exercised in drawing causal inferences and generalizing the findings to the larger population of Asian Americans. Being part of a large initiative, the measures of oral health and dental care used in the AAQoL survey were brief. For example, oral health status was assessed according to a single self-reported item. Although empirical studies support the validity of a single-item measure of self-reported oral health, ${ }^{11,12}$ future research should incorporate more-refined measures of oral health, including examinations by trained professionals. Oral pain and use of emergency dental services should also be considered as indicators of dental care need and service use. Other variables worthy of consideration would be cultural beliefs about oral health, family network and support, and health behaviors (e.g., smoking, drinking). Given the influence of culture on the way that people perceive their health needs, seek medical care, and mobilize social support, ${ }^{27}$ more attention should be paid to the potential ethnic and cultural variations. Furthermore, consideration should be given to system-level variables because individuals' oral health status and dental care use vary according to state and the area in which one lives. ${ }^{28}$ It is also important to pay attention to the availability of culturally and linguistically competent dental care professionals in local dental service environments.

Despite these limitations, the present study sheds light on the importance of using culturally and linguistically sensitive approaches to reach out to the Asian-American population. Furthermore, these findings provide ideas for interventions with respect to subgroups to be prioritized. Given the importance of immigration-specific factors, ways to provide older Asian Americans in an early stage of immigration or with cultural and linguistic barriers with access to dental care should be sought. Efforts may include education and outreach programs for older adults and their families, financial support programs for those ineligible for federal health insurance programs, and language assistance and navigation services.

\section{Acknowledgments}

Conflict of Interest: This work was supported in part by a grant from the National Institute on Aging (R01AG047106- PI: Yuri Jang, Ph.D.). The support for data collection was provided by the City of Austin's Asian American Quality of Life initiative (Contract No. 26-8275-39, PI-Yuri Jang, Ph.D.).

\section{References}

1. U.S. Department of Health and Human Services. Oral Health in America: A Report of the Surgeon General. Rockville: U.S. DHHS, National Institute of Dental and Craniofacial Research, National Institutes of Health; 2000.

2. Bloom B, Simile CM, Adams PF, et al. Oral health status and access to oral health care for U.S. adults aged 18-64: National Health Interview Survey, 2008. Vital Health Stat. 2012; 10253:1-22. 
3. Centers for Disease Control and Prevention. [Accessed September 5, 2016] Oral health: preventing cavities, gum disease, tooth loss, and oral cancers [on-line]. Available at http://www.cdc.gov/ chronicdisease/resources/publications/aag/pdf/2011/oral-health-aag-pdf-508.pdf

4. Rouxel P, Tsakos G, Chandola T, et al. Oral health-a neglected aspect of subjective well-being in later life. J Gerontol B Psychol Sci Soc Sci. 2016 Epub ahead of print.

5. Institute of Medicine. Advancing Oral Health in America. Washington, DC: National Academy of Sciences; 2011.

6. Dolan TA, Atchison K, Huynh TN, et al. Access to dental care among older adults in the United States. J Dent Educ. 2005; 69:961-974. [PubMed: 16141082]

7. Lamster IB. Oral health care services for older adults: A looming crisis. Am J Public Health. 2004; 94:699-701. [PubMed: 15117682]

8. Christian B, Chattopadhyay A, Kingman A, et al. Oral health care services utilization in the adult US population: Medical Expenditure Panel Survey 2006. Community Dent Health. 2013; 30:161167. [PubMed: 24151790]

9. Wilson F, Wang Y, Stimpson JP, et al. Use of dental services by immigration status in the United States. J Am Dent Assoc. 2016; 147:162-169. [PubMed: 26562731]

10. Clarke W, Periam C, Zoitopoulos L. Oral health promotion for linguistically and culturally diverse populations: Understanding the local non-English-speaking population. Health Educ J. 2009; 68:119-129.

11. Wu B. Differences in self-reported oral health among community-dwelling black, Hispanic, and white elders. J Aging Health. 2011; 23:267-288. [PubMed: 20858912]

12. Arcury TA, Chen H, Savoca MR, et al. Ethnic variation in oral health and social integration among older rural adults. J Appl Gerontol. 2011; 32:302-323. [PubMed: 23788829]

13. Quandt SA, Chen H, Bell RA, et al. Disparities in oral health status between older adults in a multiethnic rural community: The Rural Nutrition and Oral Health Study. J Am Geriatr Soc. 2009; 57:1369-1375. [PubMed: 19563519]

14. Bloom B, Black LI. Health of non-Hispanic Asian adults: United States, 2010-2014. NCHS Data Brief. 2016; 247:1-8.

15. Chevarley, FM. Statistical brief \#282. Rockville, MD: Agency for Healthcare Research and Quality; 2010. Percentage of Persons Unable to Get or Delayed in Getting Needed Medical Care, Dental Care, or Prescription Medicines: United States, 2007.

16. Qui Y, Ni H. Utilization of dental care services by Asians and native Hawaiian or other Pacific Islanders: United States, 1997-2000. Adv Data. 2003; 336:1-11.

17. Pew Research Center. [Accessed September 8, 2016] The rise of Asian Americans. Pew research center. [on-line]. Available at http://www.pewsocialtrends.org/files/2013/04/Asian-Americansnew-full-report-04-2013.pdf

18. Jang Y, Yoon H, Park NS, et al. Health vulnerability of immigrants with limited English proficiency: A study of older Korean Americans. J Am Geriatr Soc. 2016; 64:1498-1502. [PubMed: 27305524]

19. Jang Y, Yoon H, Park NS, et al. Dental care utilization and unmet dental needs in older Korean Americans. J Aging Health. 2014; 26:1047-1059. [PubMed: 24925691]

20. Shelley D, Russell S, Parikh NS, et al. Ethnic disparities in self-reported oral health status and access to care among older adults in NYC. J Urban Health. 2011; 88:651-662. [PubMed: 21850607]

21. National Asian Pacific Center on Aging. Asian Americans and Pacific Islanders in the United States aged 65 years and older: population, nativity, and language. Data brief. 1(3) [Accessed September 8, 2016] [on-line]. Available at http://napca.org/wp-content/uploads/2013/10/65+population-report-FINAL.pdf.

22. [Accessed September 5, 2016] City of Austin. Resolution No. 20131024-056. [on-line]. Available at http://www.austintexas.gov/edims/document.cfm?id=200017

23. Alegria M, Takeuchi D, Canino G, et al. Considering context, place, and culture: The National Latino and Asian American Study. Int J Methods Psychiatr Res. 2004; 13:208-220. [PubMed: 15719529] 
24. Pandya, C., McHugh, M., Batalova, J. Limited English Proficient Individuals in the United States: Number, Share, Growth, and Linguistic Diversity. Washington, DC: Migration Policy Institute; 2011.

25. Centers for Medicare and Medicaid Services. [Accessed September 5, 2016] Is my test, item, or service covered?. [on-line]. Available at http://www.medicare.gov/coverage/is-your-test-item-orservice-covered.html/

26. McGinn-Shapiro M. Medicaid coverage of adult dental services. State Health Policy Monitor. 2008; 2:1-6.

27. Smith A, MacEntee MI, Beattie BL, et al. The influence of culture on the oral health-related beliefs and behaviours of elderly Chinese immigrants: A meta-synthesis of the literature. J Cross Cult Gerontol. 2013; 28:27-47. [PubMed: 23397228]

28. American Dental Association. [Accessed November 27, 2016] The oral health care system: a stateby-state analysis. [on-line]. Available at http://www.ada.org/ /media/ADA/Science\%20and \%20Research/HPI/OralHealthCare-StateFacts/Oral-Health-Care-System-Full-Report.pdf 


\section{Table 1}

Descriptive Characteristics of Overall Sample $(\mathrm{N}=\mathbf{5 3 3})$

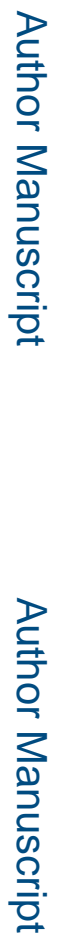

\begin{tabular}{|c|c|}
\hline Characteristic & $\%$ \\
\hline \multicolumn{2}{|l|}{ Background variable } \\
\hline \multicolumn{2}{|l|}{ Age } \\
\hline $60-74$ & 56.1 \\
\hline$\geq 75$ & 43.9 \\
\hline \multicolumn{2}{|l|}{ Sex } \\
\hline Male & 44.6 \\
\hline Female & 55.4 \\
\hline \multicolumn{2}{|l|}{ Ethnicity } \\
\hline Chinese & 30.4 \\
\hline Asian Indian & 18.4 \\
\hline Korean & 18.4 \\
\hline Vietnamese & 21.4 \\
\hline Other Asian & 11.4 \\
\hline \multicolumn{2}{|l|}{ Marital status } \\
\hline Married & 74.7 \\
\hline Not married & 25.3 \\
\hline \multicolumn{2}{|l|}{ Education } \\
\hline$\geq$ High school graduation & 64.5 \\
\hline$<$ High school graduation & 35.5 \\
\hline \multicolumn{2}{|l|}{ Employed full time } \\
\hline Yes & 13.7 \\
\hline No & 86.3 \\
\hline \multicolumn{2}{|l|}{ Financial status } \\
\hline Able to make ends meet & 70.1 \\
\hline Not able to make ends meet & 29.9 \\
\hline \multicolumn{2}{|c|}{ Length of stay in the United States, years } \\
\hline$\geq 10$ & 80.2 \\
\hline$<10$ & 19.8 \\
\hline \multicolumn{2}{|l|}{ English proficiency } \\
\hline Proficient & 16.3 \\
\hline Limited & 83.7 \\
\hline \multicolumn{2}{|l|}{ Dental health Insurance } \\
\hline Yes & 38.7 \\
\hline No & 61.3 \\
\hline \multicolumn{2}{|l|}{ Self-rated oral health } \\
\hline Excellent, very good, good & 54.9 \\
\hline Fair, poor & 45.1 \\
\hline
\end{tabular}

J Am Geriatr Soc. Author manuscript; available in PMC 2017 October 11. 


\begin{tabular}{|c|c|c|}
\hline \multirow{4}{*}{ 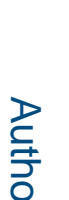 } & Characteristic & $\%$ \\
\hline & \multicolumn{2}{|c|}{ Use of preventive dental care services } \\
\hline & Yes & 55.5 \\
\hline & No & 44.5 \\
\hline
\end{tabular}


Table 2

\section{Logistic Regression Models of Oral Health and Dental Care Outcomes}

\begin{tabular}{|c|c|c|c|}
\hline & $\begin{array}{l}\text { No Dental Health } \\
\text { Insurance }\end{array}$ & $\begin{array}{c}\text { Poor or Fair Self-Rated } \\
\text { Oral Health }\end{array}$ & $\begin{array}{l}\text { No Use of Preventive } \\
\text { Dental Care Services }\end{array}$ \\
\hline Characteristic & \multicolumn{3}{|c|}{ Odds Ratio (95\% Confidence Interval) } \\
\hline \multicolumn{4}{|l|}{ Background variable } \\
\hline Age $\geq 75$ & $1.65(1.01-2.72)^{a}$ & $1.39(0.88-2.23)$ & $0.85(0.51-1.40)$ \\
\hline Female & $1.38(0.86-2.23)$ & $0.70(0.44-1.11)$ & $0.94(0.58-1.52)$ \\
\hline \multicolumn{4}{|l|}{ Ethnicity (reference Chinese) } \\
\hline Asian Indian & $2.53(1.18-5.42)^{a}$ & $0.53(0.29-1.05)$ & $1.12(0.54-2.28)$ \\
\hline Korean & $1.36(0.70-2.66)$ & $0.99(0.53-1.83)$ & $0.82(0.42-1.59)$ \\
\hline Vietnamese & $1.16(0.58-2.32)$ & $0.38(0.19-0.76)^{b}$ & $1.73(0.85-3.51)$ \\
\hline Other Asian & $0.81(0.35-1.86)$ & $0.54(0.23-1.25)$ & $2.03(0.87-4.72)$ \\
\hline Not married & $1.52(0.86-2.69)$ & $1.06(0.62-1.83)$ & $1.17(0.68-2.02)$ \\
\hline Education $<$ high school graduation & $1.45(0.85-2.47)$ & $1.66(1.02-2.72)^{a}$ & $1.08(0.65-1.81)$ \\
\hline Not employed full time & $2.14(1.12-4.11)^{a}$ & $1.12(0.56-2.24)$ & $0.81(0.40-1.63)$ \\
\hline Not able to make ends meet & $2.26(1.30-3.94)^{b}$ & $2.81(1.71-4.60)^{c}$ & $1.00(0.59-1.69)$ \\
\hline Length of stay in the United States $<10$ years & $5.87(2.69-12.7)^{\mathcal{C}}$ & $1.55(0.89-2.73)$ & $3.96(2.12-7.42)^{c}$ \\
\hline Limited English proficiency & $3.47(1.75-6.87)^{c}$ & $3.19(1.49-6.81)^{b}$ & $0.99(0.49-2.00)$ \\
\hline No dental health insurance & - & $1.48(0.90-2.45)$ & $6.42(3.69-11.2)^{c}$ \\
\hline Poor or fair self-rated oral health & - & - & $1.50(0.88-2.38)$ \\
\hline \multicolumn{4}{|l|}{ Summary statistics } \\
\hline$-2 \log$ likelihood & 453.0 & 483.5 & 454.5 \\
\hline Chi-square (degrees of freedom) & $119.0(12)^{c}$ & $92.6(13)^{c}$ & $111.2(14)^{c}$ \\
\hline
\end{tabular}

${ }^{a} P<.05$,

$b_{.01}$

$c_{.001}$ 